\title{
Fatores de adesão e permanência de idosos com diabetes tipo 2 a um programa de exercício físico
}

\author{
Factors influencing the initiation and continuation of physical \\ exercise programs by elderly individuals with type 2 diabetes
}

Iago Vilela Dantas ${ }^{1}$

Gabriela Nascimento Lima ${ }^{2}$

Ana Patrícia Siqueira Tavares Falcão ${ }^{3}$

Denise Maria Martins Vancea ${ }^{4}$

Dario Celestino Sobral Filho ${ }^{5}$

Endereço para Correspondência:

Iago Vilela Dantas

Rua Arnóbio Marquês, 310 - Santo Amaro

50100-130 - Recife, PE [Brasil]

iagovilelad@gmail.com

\footnotetext{
1 Programa de Pós-Graduação em Educação Física Universidade Federal de Pernambuco - UFPE. Recife, PE Brasil.

ORCID: https://orcid.org/0000-0002-5502-3889

2 Escola Superior de Educação Física - Universidade de Pernambuco - UPE. Recife, PE - Brasil ORCID: https://orcid.org/0000-0003-4790-2397

3 Escola Superior de Educação Física - Universidade de Pernambuco - UPE. Recife, PE - Brasil ORCID: https://orcid.org/0000-0002-1953-1042

4 Escola Superior de Educação Física - Universidade de Pernambuco - UPE. Recife, PE - Brasil ORCID: https://orcid.org/0000-0002-0590-8937

5 Faculdade de Ciências Médicas - Universidade de Pernambuco - UPE. Recife, PE - Brasil. ORCID: https://orcid.org/0000-0002-5301-7741
}

\begin{abstract}
Resumo
Introdução: Grande parte dos idosos com diabetes tipo 2 não praticam exercício físico regular. Objetivos: Identificar os fatores que influenciam idosos com diabetes tipo 2 a praticarem exercícios físicos. Métodos: Trata-se de um estudo descritivo e analítico, transversal quantitativo. A amostra foi composta por 49 idosos, com diagnóstico de diabetes tipo 2. Foram aplicados questionários sobre adesão, permanência e desistência ao exercício e sintomas depressivos. Para análise dos dados foi calculado o alpha de Cronbach e foi realizada análise fatorial e correlação dos dados. Resultados: Os motivos para aderir a um programa de exercício físico foram indicação de amigos e familiares, intenção de melhorar a autoestima ou a autoimagem, indicação médica; e os motivos para permanecer ativo foram aliviar tensão, fazer amigos, desenvolver competências, queimar calorias, manter a flexibilidade. Conclusão: Os fatores extrínsecos são os que mais influenciaram idosos com diabetes mellitus tipo 2 a aderir a um programa de exercícios físico e a se manterem na prática.
\end{abstract}

Descritores: Exercício. Diabetes mellitus. Idoso. Motivação.

\begin{abstract}
Introduction: A great proportion of elderly people with type 2 diabetic do not regularly practice any type of physical exercise. Objectives: To identify factors that influence elderly people with type 2 diabetes to exercise. Methods: A descriptive and analytical cross-sectional quantitative study was conducted. The sample consisted of 49 elderly individuals diagnosed with type 2 diabetes. Questionnaires were applied regarding beginning, continuing and quitting exercise programs, as well as depressive symptoms. For the data analysis, Cronbach's alpha was calculated, and factor analysis and correlation of the data were performed. Results: The following reasons for beginning a physical exercise program were discovered: encouragement by friends and family, desire to improve self-esteem or self-image and medical referral. The reasons for staying active were to relieve tension, make friends, develop skills, obtain self-reward, burn calories and maintain flexibility. Conclusion: The results showed that extrinsic factors are the ones that most influence elderly people with type 2 diabetes to begin a physical exercise program and to continue with it.
\end{abstract}

Keywords: Exercise. Diabetes mellitus. Elderly. Motivation. 


\section{Introdução}

O número de pessoas com diabetes mellitus (DM) cresce mundialmente. Estima-se que mais de 415 milhões de pessoas no mundo, entre 20 e 79 anos, possuem DM; e no Brasil, as estimativas apontam para 14,3 milhões ${ }^{1}$.

Uma possível causa do aumento da diabetes mellitus tipo 2 (DM2) é o aumento do envelhecimento, pois há grande associação da DM2 com o avanço da idade ${ }^{2}$. A população brasileira está envelhecendo, $11 \%$ dos indivíduos têm mais de 60 anos $^{3}$, e 4,809 milhões de idosos entre 60 e 79 anos possuem $\mathrm{DM}^{1}$.

O envelhecimento ocasiona grande decréscimo nas capacidades físicas. É comum o surgimento de osteoartroses, a diminuição do equilíbrio corporal, da força e da amplitude de movimento e a redução da massa óssea, o que causam limitações às atividades de vida diária do idoso, comprometendo sua qualidade de vida, tornando-o mais frágil e dependente. Essas perdas ainda são maiores quando o envelhecimento é associado à inatividade física ${ }^{4}$. O declínio da capacidade física contribui com um declínio físico e cognitivo e esses fatores podem afetar ou serem afetados pelo DM, o que explica o fato de diabéticos terem depressão duas vezes mais do que indivíduos sem essa patologia ${ }^{5}$.

Devido a esses fatores, o exercício físico torna-se necessário para os idosos, com o objetivo de diminuir a gordura ${ }^{6}$, aumentar a densidade óssea, reduzir os níveis de queda ${ }^{7,8}$, aumentar a força muscular ${ }^{9}$, diminuir os riscos de doença cardiovascular (DCV), pressão arterial (PA) média e frequência cardíaca (FC) de repouso ${ }^{10} \mathrm{e}$ aumentar a flexibilidade ${ }^{11}$. Além disso, o exercício faz-se necessário no tratamento da DM por reduzir a glicemia capilar, a de jejum e a hemoglobina glicada (HbA1c); aumentar a captação de glicose no músculo, melhorar o controle glicêmico e a sensibilidade à insulina ${ }^{12}$.

Por mais que se conheçam os benefícios do exercício físico, boa parte dos idosos com diabetes tipo 2 não pratica nenhum exercício físico regular $^{13}$, pois para se manter ativo na prática são necessários altos índices de motivação. A teoria da autodeterminação (TAD) ${ }^{14}$ expõe que a motivação é contínua, sendo caracterizada por níveis de autodeterminação que são a motivação intrínseca, que está relacionada ao prazer e a satisfação interna em realizar uma determinada ação; a extrínseca, quando a atividade é realizada como uma obrigação; e amotivação, quando o indivíduo não quer realizar a ação, executandoa de forma desorganizada, sendo acompanhada por sentimentos como frustração e medo ${ }^{14}$.

A prática do exercício físico é influenciada por fatores de natureza positiva e negativa. Os positivos são chamados de fatores facilitadores, que se caracterizam por auxiliar a prática de exercícios físicos, como gostar da atividade realizada ou ter uma indicação médica. Já os negativos, os que atrapalham ou dificultam essa prática são chamados de barreiras, que podem ser internas, referentes ao pensamento individual, ao constrangimento; ou externas, relacionadas às imposições da sociedade, ao trabalho e distância do local da prática ${ }^{14}$.

Por conseguinte, por já se ter o conhecimento da importância da prática do exercício físico para indivíduos com DM e da pouca adesão dessa população a essa prática, torna-se importante conhecer as barreiras e entender os motivos da adesão e permanência que estão relacionados a práticas do exercício físico por idosos com DM, para assim, buscar formas de contornar essas dificuldades, aumentando tanto a adesão quanto à aderência dessa população. Desse modo, objetivou-se neste estudo identificar os fatores de adesão, permanência e desistência de idosos com diabetes mellitus à prática de exercícios físicos, bem como se esses fatores são de natureza intrínseca ou extrínseca.

\section{Material e métodos}

Este estudo descritivo, analítico e transversal quantitativo foi aprovado pelo Comitê de Ética em Pesquisa em Seres Humanos 
(CEP) - Complexo Hospitalar HUOC/PROCAPE, com o número de parecer 611.627, e CAAE 25046614.9.0000.5192. Foi assinada uma carta de anuência pela Coordenação da Escola Superior de Educação Física - ESEF, da Universidade de Pernambuco - UPE, e um Termo de Autorização de Setor pela Coordenação do Programa de Exercício Físico Supervisionado para Diabéticos - realizado na ESEF, permitindo a coleta.

\section{Localização do estudo}

O estudo foi realizado na Escola Superior de Educação Física - ESEF/UPE no Doce Vida Programa de Exercícios Físicos Supervisionados para Diabéticos, existente na ESEF desde 2010. Nesse local, três vezes por semana, ocorre prática de exercícios físicos de alongamentos e treinamentos aeróbio, resistido e combinado. Em todos os dias de projeto é realizada a aferição da glicemia capilar, da pressão arterial (PA) e da frequência cardíaca (FC), antes e após cada sessão de treino. Periodicamente (trimestralmente) são realizadas avaliações físicas, coleta de glicemia de jejum e pós-prandial e palestras educativas.

\section{Participantes}

A seleção dos participantes foi realizada por conveniência e a amostra, composta por indivíduos que se enquadraram aos seguintes critérios de inclusão: apresentar diagnóstico de DM; ter 60 anos ou mais quando iniciou no programa; ter frequentado o Doce Vida por mais de três meses, ou ter participado por no mínimo um mês e ter saído do programa a mais de um mês.

\section{Metas e fases de desenvolvimento do estudo}

Foi ministrada uma pequena palestra para os idosos com DM, a fim de explicar a pesquisa a ser realizada. Foram reservados alguns dias para a coleta dos dados (aplicação dos questionários) e a assinatura do Termo de Consentimento Livre e Esclarecido (TCLE). Em relação aos indivíduos egressos, foram realizadas ligações telefônicas para convidá-los a participar do estudo.

\section{Instrumentos da pesquisa}

Aplicou-se um questionário sobre dados pessoais e socioeconômicos juntamente com um sobre os motivos de adesão à prática do exercício físico. Para os integrantes que frequentaram o programa, por no mínimo três meses, foi aplicado o questionário sobre Motivos para o Exercício que foi traduzido do Exercise Motivations Inventory-II (EMI-II) ${ }^{15}$, sobre motivos de permanência a prática do exercício físico. Já com os egressos foi aplicado o questionário sobre os motivos que influenciaram a desistência do programa, sendo considerada a desistência do programa ausência de três meses.

Os questionários de adesão e dos egressos foram desenvolvidos para este estudo, baseados em resultados de trabalhos publicados na literatura ${ }^{16-18}$.

Foi aplicada também a Escala de Depressão de Beck II (BDI-II) $)^{19}$ em todos os integrantes para verificar a presença de sintomas depressivos e a relação com a permanência ou desistência do programa. O BDI-II avalia os sintomas depressivos em quatro níveis: sem depressão, disforia, depressão moderada a grave e depressão grave.

\section{Anólise estatística}

Foi realizada uma análise descritiva dos dados por meio da distribuição de frequência. Posteriormente, foi calculado o coeficiente alfa de Cronbach objetivando analisar a consistência interna dos questionários aplicados, seguido da análise fatorial com o intuito de verificar as variáveis que mais influenciam os motivos de adesão, permanência e desistência em programas de exercício físico, bem como a correlação dos motivos com os fatores socioeconômicos. Os dados foram analisados no Programa SPSS versão 10.0 . 


\section{Resultados}

Dos 62 diabéticos que integravam o programa inicialmente, 30 indivíduos se retiraram e foram eleitos para compor a amostra dos egressos. Concomitantemente, 25 participantes se juntaram aos 32 remanescentes e, destes, 30 foram eleitos para compor a amostra dos ativos, totalizando 60 idosos selecionados para a coleta. Após perdas amostrais, apenas 49 dos 60 idosos foram incluídos no estudo, sendo 29 ativos e 20 egressos (Figura 1).

No momento da coleta, dentre os 29 participantes do grupo ativo, 16 encontravam-se no programa há pelo menos 12 meses, 8 há 6 meses e 5 há 3 meses. Dos 20 desistentes, 9 abandonaram o programa há pelo menos 6 meses, 7 há 3 meses e 4 há pelo menos 1 mês. As coletas com os idosos ativos foram realizadas em um dos dias de intervenção do Programa Doce Vida, com os egressos foram marcados horários, por meio de ligações telefônicas, para responderem os questionários na ESEF. As características gerais da amostra se encontram na Tabela 1.

Inicialmente foi testada a confiabilidade dos três questionários por meio do índice Alpha de Cronbach. O primeiro questionário, o de adesão ao exercício físico, obteve um $\alpha$ de 0,77 indicando que teve uma confiabilidade aceitável. O segundo questionário, o de permanência no exercício físico, obteve um $\alpha$ de 0,93 indicando que teve excelente confiabilidade. $\mathrm{O}$ terceiro questionário, o de desistência, obteve um $\alpha$ de 0,32 sendo este valor considerado inaceitável para esse tipo de análise. Foi realizada também uma distribuição de frequência dos dados dos questionários, separadas por grupo (Tabelas 2 e 3).

Foi desenvolvida a análise fatorial nos questionários de adesão e permanência. No questionário de adesão, 5 das 14 perguntas apresentaram maior peso na análise fatorial (Tabela 2). No questionário de permanência, 8 das 47 questões obtiveram maior peso na análise fatorial (Tabela 3). Foi realizada uma correlação das questões que

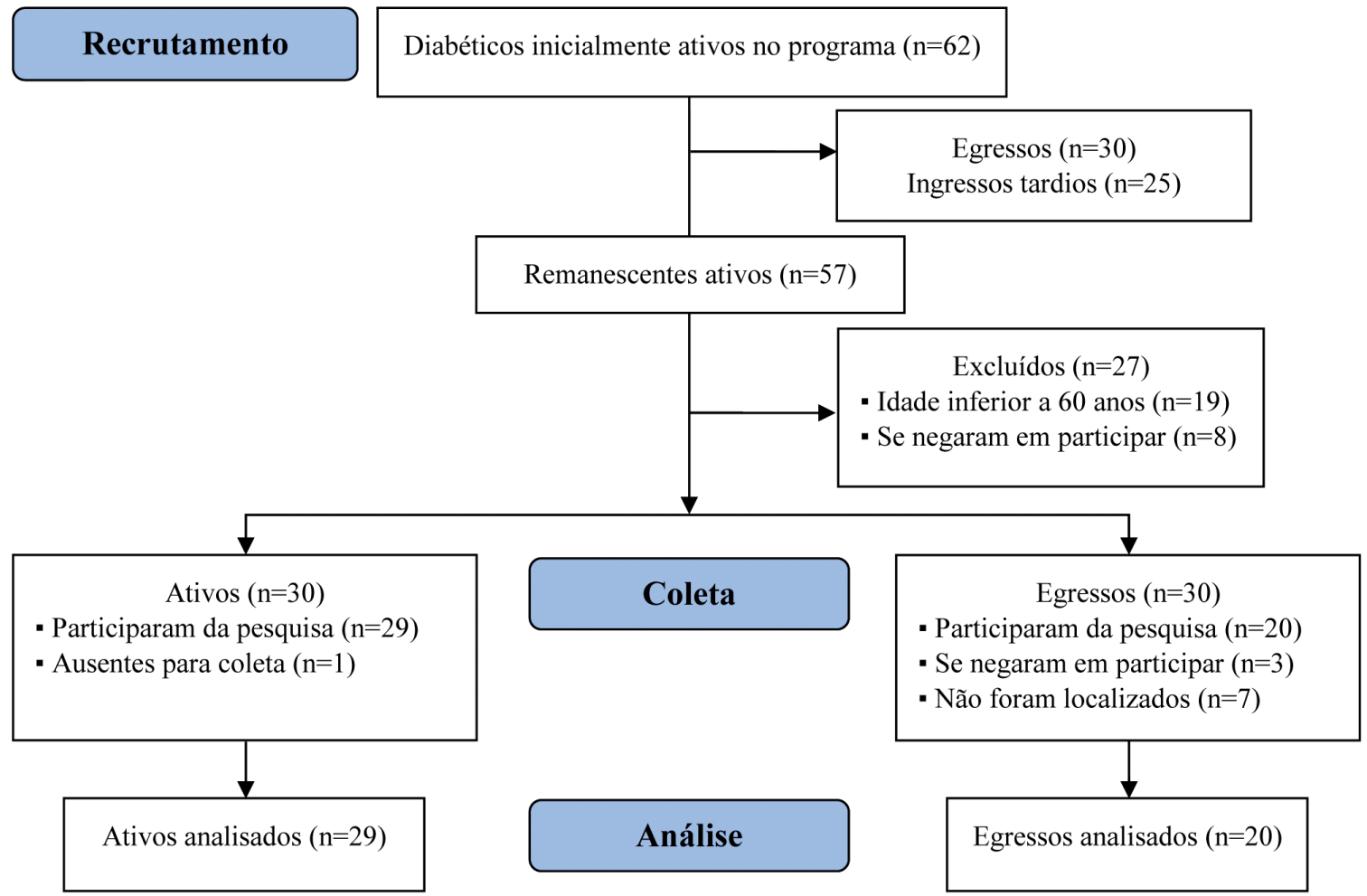

Figura l: Fluxograma amostral

Fonte: Os autores, 2019. 
Tabela 1: Caracterização geral da amostra

\begin{tabular}{|c|c|c|}
\hline $\begin{array}{l}\text { Características } \\
\mathrm{n}\end{array}$ & $\begin{array}{c}\text { Ativos } \\
29\end{array}$ & $\begin{array}{l}\text { Egressos } \\
20\end{array}$ \\
\hline \multicolumn{3}{|l|}{ Gênero } \\
\hline Feminino & $25(86,2 \%)$ & 19 (95\%) \\
\hline Masculino & $4(13,8 \%)$ & $1(5 \%)$ \\
\hline Idade & $70,8 \pm 6,1$ & $71,8 \pm 5,7$ \\
\hline \multicolumn{3}{|l|}{ Escolaridade } \\
\hline Analfabetos & $2(6,9 \%)$ & $2(10 \%)$ \\
\hline Ensino Fundamental Incompleto & $12(41,4 \%)$ & $5(25 \%)$ \\
\hline Ensino Fundamental Completo & $2(6,9 \%)$ & $3(15 \%)$ \\
\hline Ensino Médio Completo & $7(24,1 \%)$ & $4(20 \%)$ \\
\hline Ensino Superior Completo & $6(20,7 \%)$ & $6(30 \%)$ \\
\hline \multicolumn{3}{|l|}{ Renda } \\
\hline$\leq 1$ Salário mínimo & $3(10,3 \%)$ & $3(15 \%)$ \\
\hline >1 a 2 Salários mínimos & $10(34,5 \%)$ & $6(30 \%)$ \\
\hline >2 a 4 Salários mínimos & $12(41,4 \%)$ & $7(35 \%)$ \\
\hline >4 Salários mínimos & $4(13,8 \%)$ & $4(20 \%)$ \\
\hline \multicolumn{3}{|c|}{ Medicamentos para diabetes } \\
\hline Sem medicamentos & $1(3,4)$ & 0 \\
\hline Hipoglicemiante oral & $16(55,2 \%)$ & $15(75 \%)$ \\
\hline Insulina & $3(10,3 \%)$ & $3(15 \%)$ \\
\hline Hipoglicemiante oral e insulina & $9(31,1 \%)$ & $2(10 \%)$ \\
\hline $\begin{array}{l}\text { Tempo de diagnóstico de } \\
\text { diabetes }\end{array}$ & $16,3 \pm 9,5$ & $15,7 \pm 9,2$ \\
\hline \multicolumn{3}{|l|}{ Doenças psicológicas } \\
\hline Não & $26(87,9 \%)$ & $18(90 \%)$ \\
\hline Ansiedade & $2(6,9 \%)$ & 0 \\
\hline Depressão & $1(3,4 \%)$ & $2(10 \%)$ \\
\hline \multicolumn{3}{|l|}{ Antidepressivo } \\
\hline Não usa & $27(93,1 \%)$ & $18(90 \%)$ \\
\hline Usa & $2(6,9 \%)$ & $2(10 \%)$ \\
\hline \multicolumn{3}{|l|}{ Tipo de treinamento } \\
\hline Aeróbio & $4(13,8 \%)$ & $4(10 \%)$ \\
\hline Resistido & $10(34,5 \%)$ & $9(45 \%)$ \\
\hline Combinado & $5(17,2 \%)$ & $5(25 \%)$ \\
\hline Relaxamento & $10(34,5 \%)$ & $2(10 \%)$ \\
\hline \multicolumn{3}{|c|}{$\begin{array}{l}\text { Análise de distribuição de frequência, média e } \\
\text { desvio-padrão. }\end{array}$} \\
\hline & & \\
\hline
\end{tabular}

tiveram um maior peso pela análise fatorial com os dados socioeconômicos (renda, escolaridade e estado civil). Não houve correlação com os motivos de aderência. Contudo, observou-se uma correlação moderada com uma questão do questionário de permanência (manter a flexibilidade dos músculos) com renda $(0,54)$ e escolaridade $(0,56)$.
Quanto aos sintomas depressivos avaliados pelo $\mathrm{BDI}-\mathrm{II}^{20}$, foi observado que $63,2 \%$ da amostra encontravam-se sem sintomas depressivos (38,8\% - ativos e $24,4 \%$ - egressos); $16,3 \%$, com disforia (10,2\% - ativos e 6,1\% - egressos); $12,2 \%$ apresentaram sintomas de depressão moderada $(4,1 \%$ ativos e $8,1 \%$ - egressos) e $8,1 \%$, com sintomas de depressão grave (6,1\% - ativos e $2 \%$ - egressos).

Foi realizada também uma correlação dos sintomas depressivos com os principais motivos dos questionários de adesão e permanência segundo a análise fatorial com os fatores socioeconômicos. Quando os sintomas depressivos foram correlacionados com os questionários de adesão e de permanência, foi observada uma correlação forte apenas com o de permanência, cujo motivo foi manter a flexibilidade dos músculos $(0,61)$. Quando os sintomas depressivos foram analisados com os dados socioeconômicos, não foi observada correlação com o grupo de adesão. Com o grupo de permanência, podese observar uma correlação moderada negativa com a escolaridade $(-0,54)$ e com a renda $(-0,4)$.

Referente aos motivos para desistência, o questionário apresentou um $\alpha$ de Cronbach inaceitável, não sendo possível identificar os principais motivos de abandono do programa, o que inviabilizou também o cálculo da análise fatorial. Pode-se destacar que uma das dificuldades encontradas para a análise foram as respostas que se apresentaram de forma binária, não se enquadrando na escala proposta (tipo Likert).

\section{Discussão}

Os achados demonstraram que os fatores de maior influência para adesão ao programa de exercício físico se originaram de motivação extrínseca, sendo eles indicação de amigos e familiares, indicação médica e não tomar insulina, assim como também apresentaram influências intrínsecas, como melhorar a autoestima e a autoimagem (Tabela 2). Quanto à permanência no programa, com exceção do motivo de o exercício ser gratificante, a grande influência permane- 
Tabela 2: Distribuição de frequência e anólise fatorial dos principais motivos de adesão ao exercício físico

\begin{tabular}{|c|c|c|c|c|c|c|c|c|c|c|c|c|c|}
\hline \multirow{2}{*}{ Motivos } & \multicolumn{2}{|c|}{0} & \multicolumn{2}{|c|}{1} & \multicolumn{2}{|c|}{2} & \multicolumn{2}{|c|}{3} & \multicolumn{2}{|c|}{4} & \multicolumn{2}{|c|}{5} & \multirow{2}{*}{$r$} \\
\hline & $\mathrm{f}$ & $\%$ & $f$ & $\%$ & $f$ & $\%$ & $f$ & $\%$ & $f$ & $\%$ & f & $\%$ & \\
\hline $\begin{array}{c}\text { Indicação de amigos e } \\
\text { familiares }\end{array}$ & 12 & 24,5 & 0 & 0,0 & 1 & 2,0 & 5 & 10,2 & 3 & 6,1 & 28 & 57,1 & 0,92 \\
\hline Melhorar a autoestima & 5 & 10,2 & 0 & 0,0 & 2 & 4,1 & 9 & 18,4 & 5 & 10,2 & 28 & 57,1 & 0,90 \\
\hline Indicação médica & 16 & 32,7 & 1 & 2,0 & 0,0 & 0,0 & 4 & 8,2 & 3 & 6,1 & 25 & 51,0 & 0,89 \\
\hline Melhorar a autoimagem & 9 & 18,4 & 1 & 2,0 & 1 & 2,0 & 8 & 16,3 & 2 & 4,1 & 28 & 57,1 & 0,88 \\
\hline Não tomar insulina & 43 & 87,8 & 0 & 0,0 & 0 & 0,0 & 0 & 0,0 & 0 & 0,0 & 6 & 12,2 & 0,87 \\
\hline
\end{tabular}

f: distribuição de frequência dos motivos; \%: valor em porcentagem da distribuição de frequência; $r$ fator de correlação. Fonte: Os autores, 2019.

Tabela 3: Distribuição de frequência e anólise fatorial dos principais motivos de permanência ao exercício físico

\begin{tabular}{|c|c|c|c|c|c|c|c|c|c|c|c|c|c|}
\hline \multirow{2}{*}{ Motivos } & \multicolumn{2}{|c|}{0} & \multicolumn{2}{|c|}{1} & \multicolumn{2}{|c|}{2} & \multicolumn{2}{|c|}{3} & \multicolumn{2}{|c|}{4} & \multicolumn{2}{|c|}{5} & \multirow[b]{2}{*}{ r } \\
\hline & $f$ & $\%$ & $f$ & $\%$ & f & $\%$ & $f$ & $\%$ & $f$ & $\%$ & $f$ & $\%$ & \\
\hline Aliviar a tensão & 1 & 3,4 & 0 & 0,0 & 4 & 13,8 & 4 & 13,8 & 3 & 10,3 & 17 & 58,6 & 0,92 \\
\hline Fazer novos amigos & 3 & 10,3 & 2 & 6,9 & 2 & 6,9 & 3 & 10,3 & 0 & 0,0 & 19 & 65,5 & 0,89 \\
\hline $\begin{array}{l}\text { Desenvolver competências } \\
\text { pessoais }\end{array}$ & 5 & 17,2 & 1 & 3,4 & 2 & 6,9 & 5 & 17,2 & 1 & 3,4 & 15 & 51,7 & 0,88 \\
\hline $\begin{array}{l}\text { Fazer exercício é } \\
\text { gratificante }\end{array}$ & 0 & 0,0 & 0 & 0,0 & 1 & 3,4 & 1 & 3,4 & 2 & 6,9 & 25 & 86,2 & 0,85 \\
\hline $\begin{array}{c}\text { O exercício ajuda a queimar } \\
\text { calorias }\end{array}$ & 0 & 0,0 & 0 & 0,0 & 1 & 3,4 & 3 & 10,3 & 1 & 3,4 & 24 & 82,8 & 0,85 \\
\hline $\begin{array}{l}\text { Manter a flexibilidade dos } \\
\text { músculos }\end{array}$ & 0 & 0,0 & 0 & 0,0 & 2 & 6,9 & 4 & 13,8 & 2 & 6,9 & 21 & 72,4 & 0,85 \\
\hline $\begin{array}{l}\text { Conseguir coisas que os } \\
\text { outros não são capazes }\end{array}$ & 9 & 31,0 & 0 & 0,0 & 1 & 3,4 & 4 & 13,8 & 3 & 10,3 & 12 & 41,4 & 0,85 \\
\hline Ter objetivos a atingir & 2 & 6,9 & 1 & 3,4 & 0 & 0,0 & 2 & 6,9 & 2 & 6,9 & 22 & 75,9 & 0,84 \\
\hline
\end{tabular}

f: distribuição de frequência dos motivos; \%: valor em porcentagem da distribuição de frequência; $r$ fator de correlação.

Fonte: Os autores, 2019.

ceu de motivos extrínsecos, dentre eles, aliviar a tensão, fazer novos amigos, desenvolver competências pessoais, queimar calorias e manter a flexibilidade do músculo (Tabela 3). Por fim, observou-se que $20,3 \%$ da amostra possuía algum sintoma depressivo, sendo encontrada uma correlação negativa com a escolaridade e a renda no grupo de permanência, e uma positiva entre a questão manter a flexibilidade dos músculos.

Do questionário dos motivos de adesão, as questões indicação de amigos e/ou familiares e indicação médica apresentaram o quanto o aconselhamento externo influenciou na busca por um exercício físico, a primeira provavelmente por questão de cuidado e/ou companhia e a segunda pela influência e obediência que se tem pelo médico. Sobre o ponto de vista social, o principal motivo que conduziu a adesão ao programa de exercício físico foi a questão relacionamentos. A questão melhorar a autoestima reflete a crença de que se sentiriam melhor, mais confiantes com a prática do exercício físico, tanto por questões pessoais e fisiológicas quanto pela dimensão social presente. Quanto à questão melhorar a autoimagem, ela expõe o quão presente está a vaidade nos idosos analisados, pois demonstraram que se preocupam com o estereótipo e percebem que podem ter benefícios estéticos por meio do exercício físico ${ }^{21}$.

Os achados dos motivos extrínsecos na adesão de idosos diabéticos são similares a resultados prévios ${ }^{20,21}$ de investigações em que se 
constataram que, para viabilizar o exercício físico, é preciso apoio social, o que ratifica a necessidade de incentivos externos para idosos e pessoas com diabetes iniciarem a prática de exercícios físicos. Porém, alguns estudos ${ }^{16,17}$ apontaram a questão saúde como sendo o grande motivador extrínseco para iniciar a prática do exercício físico; contudo, no atual estudo, não foi possível encontrar esses resultados, talvez devido à falta de conhecimento da amostra sobre os benefícios do exercício físico para indivíduos com DM.

Concernente aos motivos intrínsecos, com exceção de um estudo que mostrou que a adesão de diabéticos pode ser melhorada estimulandose a motivação intrínseca por motivos intimamente ligados a fatores motivacionais ${ }^{22}$, os achados do estudo aqui apresentado não corroboram a literatura atual a qual vem demonstrando que os fatores extrínsecos são os principais motivos que levam idosos diabéticos a se exercitarem $^{16,17,20,21}$.

Em relação ao questionário de permanência, apenas uma das oito questões se originava da motivação intrínseca. Outros estudos também apontaram a motivação intrínseca presente nos motivos de permanência na prática do exercício físico, sendo identificado que as pessoas sentiam prazer com tal prática ${ }^{17,21}$.

Grande parte da amostra (93,1\%) afirmou que fazer exercício físico é gratificante e que isso influenciou consideravelmente ou muito a permanência na prática dessa atividade. Isto pode ser explicado por meio da $\mathrm{TAD}^{15}$, na qual se afirma que, quando uma pessoa é movida intrinsecamente, realiza uma determinada atividade porque gosta, apresentando, assim, mais chance de continuar ativa nessa prática pela satisfação e pelo prazer que sente quando a realiza.

Porém, a motivação extrínseca foi a principal responsável pela permanência no programa. Esse resultado é consistente com achados pré$\operatorname{vios}^{16,17,20,21}$ de estudos nos quais os voluntários mostraram ter noção dos benefícios que a prática do exercício físico proporciona em diversos aspectos de suas vidas fazendo com que essa cons- ciência os motive a continuar praticando-os pelos benefícios gerados e não por sentirem prazer.

Um dos principais motivos de permanência foi aliviar a tensão, que pode ser compreendido pelo fato de o exercício físico aumentar a produção de endorfinas, o que auxilia na redução da dor e promove um estado de euforia e prazer ${ }^{17,21}$.

Em relação à população idosa portadora de diabetes, existe uma problemática: a tendência ao isolamento social ${ }^{23}$. São muitas as perdas que os idosos sofrem, tanto no aspecto físico quanto no social ${ }^{4,5} \mathrm{e}$ isso remete a uma maior dependência, limitando o seu dia a dia e o convívio social. Devido a isso, participar de um grupo social é imensamente encorajador para a prática do exercício físico e disponibiliza a oportunidade de conviver com pessoas da mesma idade e com mesmo estilo de vida, o que explica a questão fazer novos amigos ter mostrado boa influência na permanência do exercício físico. Um estudo apontou que um dos principais motivos para permanecer ativo numa prática de exercício físico é o meio social ${ }^{17}$. Outra pesquisa mostrou que a maior parte dos integrantes de programas de exercícios prefere exercitar-se com um companheiro ou com um grupo do que se exercitar sozinha ${ }^{21}$. O que ratifica a necessidade que essas pessoas têm de se relacionar com outras.

Outra questão de grande importância está relacionada à perda de peso. Quando os idosos analisados afirmaram que um dos motivos que os influenciou a continuar o exercício físico foi a questão o exercício ajuda a queima de calorias, isso indicou que eles sentem a necessidade de perder peso e sabem que o exercício físico pode auxiliar nessa redução. Diversos estudos com diabéticos comprovaram que um programa de exercícios físicos pode reduzir o $\mathrm{IMC}^{6}$, e isso remete não somente a perda de peso, mas também a outros benefícios, tais como melhora das variáveis metabólicas ${ }^{12}$ e diminuição do risco cardiovascular ${ }^{10}$.

Outro benefício do exercício físico apontado é a flexibilidade. No envelhecimento há uma redução da flexibilidade ${ }^{11}$. Pessoas com DM também têm uma maior tendência a essa diminui- 
ção, pois a glicose adere a estruturas do corpo, incluindo as cartilagens e o colágeno, provocando enrijecimento das articulações e diminuição de sua flexibilidade. Para evitar essa perda é necessário manter o DM controlado e praticar exercícios físicos, pois a prática regular dessa atividade promove aumento na flexibilidade $\mathrm{e}^{11}, \mathrm{o}$ que explica o fato de manter a flexibilidade dos músculos ser um motivo de grande influência para a permanência no exercício físico.

Os três outros motivos que influenciaram a permanência no exercício físico são pouco subjetivos. Desenvolver competências pessoais remete a níveis, habilidades, experiências, aprendizados que podem ser alcançados por meio do exercício físico. Conseguir coisas que os outros não são capazes faz alusão a querer ser melhor que outra pessoa, entretanto, no contexto deste estudo, pode-se interpretar que os participantes buscam ser melhor no sentido de ter uma saúde melhor, uma maior independência, ter condições físicas que indivíduos ao seu redor não conseguem ter devido à idade e a doenças. E "ter objetivos a atingir" pode-se entender como os benefícios que o exercício físico pode promover, tais como controle do DM e redução do peso corporal.

Em relação ao $\mathrm{BDI}-\mathrm{II}^{19}$, este questionário não realiza diagnóstico de depressão, apenas indica a existência de sintomas depressivos. Por meio desse instrumento foi observado que a maior parte da amostra não apresentou sintomas depressivos (63,2\%). Em contrapartida, 20,3\% têm algum sintoma depressivo, o que corrobora a pesquisa de Trief (2007), na qual $20 \%$ dos idosos podem ter transtornos mentais, como a depressão, sem que sejam atribuídos ao envelhecimento normal, pois ocorrem diversas mudanças nos aspectos físico e psicossocial que podem acarretar a depressão e o diabetes ${ }^{5}$.

No atual estudo também se avaliou a correlação entre sintomas depressivos e a escolaridade e a renda, sendo encontrada correlação negativa dos sintomas depressivos com a escolaridade e a renda no grupo de permanência, apontando que quanto maior o nível de instrução e melhor a situação financeira menor a presença dos sin- tomas depressivos. Adicionalmente, pôde ser observada correlação positiva entre os sintomas depressivos e a questão manter a flexibilidade dos músculos, no questionário de permanência, mostrando que quanto mais os diabéticos têm sintomas depressivos mais eles sentem necessidade de continuar ativos, o que demonstra a consciência que os diabéticos têm em relação às suas limitações e aos benefícios promovidos pelo exercício físico. Quanto à correlação dos dados socioeconômicos com manter a flexibilidade dos músculos indica que quanto maior o nível de instrução e melhor for a situação financeira, maior é o cuidado e a preocupação com a manutenção da flexibilidade.

\section{Conclusão}

Neste estudo, objetivou-se identificar os fatores de adesão e permanência de idosos com diabetes mellitus à prática de exercícios físicos, bem como se esses fatores são de natureza intrínseca ou extrínseca.

Os achados demonstraram que os fatores facilitadores para a adesão ao exercício físico foram: indicação de amigos e familiares, intenção de melhorar a autoestima ou a autoimagem, indicação médica e suspensão do uso da insulina. Quando se trata dos fatores facilitadores para permanência na prática do exercício, pôde-se observar que os principais motivos foram aliviar a tensão, fazer novos amigos, desenvolver competências pessoais, queimar calorias, manter flexibilidade do músculo, conseguir coisas que os outros não são capazes, ter objetivos a atingir e o fato de o exercício físico ser gratificante para os idosos que o realiza.

Conclui-se, portanto, que para melhorar a adesão de idosos diabéticos à prática de exercício físico, assim como aumentar o grau de permanência destes indivíduos em programas que ofereçam essa prática, deve-se focar na motivação extrínseca desses sujeitos por meio de incentivos externos, companhia e estímulos pessoais. 


\section{Referências}

1. International Diabetes Federation. IDF Atlas. 7th ed. Brussels, Belgium: International Diabetes Federation. 2015.

2. Sociedade Brasileira de Diabetes. Diretrizes da Sociedade Brasileira de Diabetes. São Paulo, Brasil: Clannad. 2017-2018.

3. Instituto Brasileiro de Geografia e Estatística. Distribuição da população por sexo, segundo os grupos de idade. Censo Demográfico. 2018.

4. Veronese N, Cooper C, Reginster JY, Hochberg M, Branco J, Bruyère $\mathrm{O}$, et al. Type 2 diabetes mellitus and osteoarthritis. Semin Arthritis Rheum. 2019 Jan 11;(18):30586-9.

5. Khan ZD, Lutale J, Moledina SM. Prevalence of depression and associated factors among diabetic patients in an outpatient diabetes clinic. Psychiatry J. 2019 Jan 15;2083196.

6. Batsis JA, Gill LE, Masutani RK, Adachi-Mejia AM, Blunt HB, Bagley PJ, et al. Weight loss interventions in older adults with obesity: a systematic review of randomized controlled trials since 2005. J Am Geriatr Soc. 2017 Feb;65(2):257-68.

7. Province MA, Hadley EC, Hornbrook MC, Lipsitz LA, Miller JP, Mulrow CD, et al. The effects of exercise on falls in elderly patients. A preplanned meta-analysis of the FICSIT Trials. Frailty and Injuries: Cooperative Studies of Intervention Techniques. JAMA. 1995 May 3;273(17):1341-7.

8. Cheng P, Tan L, Ning P, Li L, Gao Y, Wu Y, et al. Comparative effectiveness of published interventions for elderly fall prevention: a systematic review and network meta-analysis. Int J Environ Res Public Health. 2018 Mar 12;15(3).

9. Sardeli AV, Komatsu TR, Mori MA, Gáspari AF, Chacon-Mikahil MPT. Resistance training prevents muscle loss induced by caloric restriction in obese elderly individuals: a systematic review and metaanalysis. Nutrients. 2018 Mar 29;10(4).

10. Rodrigues-Krause J, Farinha JB, Krause M, ReischakOliveira Á. Effects of dance interventions on cardiovascular risk with ageing: systematic review and meta-analysis. Complement Ther Med. 2016 Dec;29:16-28.

11. Bullo V, Bergamin M, Gobbo S, Sieverdes JC, Zaccaria M, Neunhaeuserer D, et al. The effects of Pilates exercise training on physical fitness and wellbeing in the elderly: a systematic review for future exercise prescription. Prev Med. 2015 Jun;75:1-11.
12. Pan B, Ge L, Xun YQ, Chen YJ, Gao CY, Han X, et al. Exercise training modalities in patients with type 2 diabetes mellitus: a systematic review and network meta-analysis. Int J Behav Nutr Phys Act. 2018 Jul 25;15(1):72.

13. Lee J. The Relationship between physical activity and dementia: a systematic review and metaanalysis of prospective cohort studies. J Gerontol Nurs. 2018 Oct 1;44(10):22-9.

14. Ryan RM, Deci EL. Intrinsic and extrinsic motivations: classic definition and new direction. Contemp Educ Psychol. 2000 Jan 25;(1):54-67.

15. Markland D, Ingledew DK. The measurement of exercise motives: factorial validity and invariance across gender of a revised Exercise Motivation Inventory. Br J Health Psychol. 1997;(2):361-76.

16. Fortier MS, Sweet SN, Tulloch H, Blanchard CM, Sigal RJ, Kenny GP, et al. Self-determination and Exercise Stages of Change: results from the diabetes aerobic and resistance exercise trial. J Health Psychol. 2012;(17):87-99.

17. Godoy RF. Benefícios do exercício físico sobre a área emocional. Movimento. 2002;8(2):7-16.

18. Carneiro RS, Falcone E, Clark C, Prette Z, Prette A. Qualidade de vida, apoio social e depressão em idosos: relação com habilidades sociais. Psicol Reflex Crit. 2007; 20(2):229-37.

19. Gomes O, Marcio H, Gorenstein C, Neto FL, Andrade LH, Wang YP. Validation of the Brazilian Portuguese version of the Beck Depression Inventory-II in a community sample. Rev Bras Psiquiatr. 2012;(4):389-94.

20. Miller ST, Marolen K. Physical activity-related experiences, counseling expectations, personal responsibility, and altruism among urban African American women with Type 2 Diabetes. Diabetes Educ. 2012;38(2):229-35.

21. Freitas CMSM, Santiago MS, Viana AT, Leão AC, Freyre C. Aspectos motivacionais que influenciam a adesão e manutenção de idosos a programas de exercícios físicos. Rev Bras Cineantropom Desempenho Hum. 2007;9(1):92-100.

22. Kellie MR. Intrinsic and extrinsic factors affecting patient engagement in diabetes self-management: perspectives of a certified diabetes educator. Clin Therap. 2013;35(2):170-8.

23. Trief PM. Depression in Elderly Diabetes Patients. Diabetes Spectrum. 2007;20(2):71-5. 